\title{
Different nucleosomal architectures at early and late replicating origins in Saccharomyces cerevisiae
}

\author{
Ignacio Soriano ${ }^{1 \dagger}$, Esther C Morafraile ${ }^{1 \dagger}$, Enrique Vázquez ${ }^{1}$, Francisco Antequera ${ }^{1}$ and Mónica Segurado ${ }^{1,2^{*}}$
}

\begin{abstract}
Background: Eukaryotic genomes are replicated during $S$ phase according to a temporal program. Several determinants control the timing of origin firing, including the chromatin environment and epigenetic modifications. However, how chromatin structure influences the timing of the activation of specific origins is still poorly understood.

Results: By performing high-resolution analysis of genome-wide nucleosome positioning we have identified different chromatin architectures at early and late replication origins. These different patterns are already established in G1 and are tightly correlated with the organization of adjacent transcription units. Moreover, specific early and late nucleosomal patterns are fixed robustly, even in rpd3 mutants in which histone acetylation and origin timing have been significantly altered. Nevertheless, higher histone acetylation levels correlate with the local modulation of chromatin structure, leading to increased origin accessibility. In addition, we conducted parallel analyses of replication and nucleosome dynamics that revealed that chromatin structure at origins is modulated during origin activation.
\end{abstract}

Conclusions: Our results show that early and late replication origins present distinctive nucleosomal configurations, which are preferentially associated to different genomic regions. Our data also reveal that origin structure is dynamic and can be locally modulated by histone deacetylation, as well as by origin activation. These data offer novel insight into the contribution of chromatin structure to origin selection and firing in budding yeast.

\section{Background}

Eukaryotic chromosomes are replicated from multiple replication origins. In budding yeast, replication origins are termed ARS (autonomously replicating sequences) and contain the ARS consensus sequence (ACS), which is essential for origin activity [1]. During the G1 phase, pre-replicative complexes (pre-RCs), composed of the origin recognition complex (ORC), Cdc6, Cdt1, and an inactive form of the replicative helicase $\mathrm{Mcm} 2-7 \mathrm{com}$ plex are assembled into ACS-containing origin regions, a process called 'licensing' [2]. During S phase, origin activation is induced by the activation of the MCM2-7 helicase and by the recruitment of further initiation factors, including Mcm10, Cdc45, GINS, Sld2, Sld3 and Dpb11, to form pre-initiation complexes (pre-IC) [3]. Although all replication origins are 'licensed' during G1

\footnotetext{
*Correspondence: monicas@usal.es

${ }^{\dagger}$ Equal contributors

'Instituto de Biología Funcional y Genómica, Consejo Superior de

Investigaciones Científicas/ Universidad de Salamanca (CSIC/USAL), Campus Miguel de Unamuno, Salamanca 37007, Spain

2Departamento de Microbiología y Genética, (USAL), Campus Miguel de Unamuno, 37007 Salamanca, Spain
}

phase $[4,5]$, not all of them are activated during the subsequent replication cycle and, moreover, origins are activated at different times along $\mathrm{S}$ phase. The temporal order of origin activation is called the replication timing program, and it is established in G1 phase [6].

The mechanisms orchestrating the timing program are not fully understood, but many studies have indicated that the firing of origins can be influenced by different factors involving replication initiation mechanisms, epigenetic modifications, chromosomal position and the chromatin context [7-17].

Limiting levels of initiation factors have been reported [15,16], and constitute a point of control of replication initiation. The amount of Sld3, Sld2, Sld7, Dpb11, Cdc45 and Dbf4 factors is below the number of "licensed" origins, and hence activation is necessarily restricted to a subset of origins. Some of these factors, i.e. Sld3 and Cdc45, can be detected specifically at some early firing origins during G1 $[11,18,19]$, which is critical for promoting their activation at the beginning of $\mathrm{S}$ phase. However, even though the sequential activation of origins can be explained by limiting levels of essential initiation proteins, an open question is why limiting factors are preferentially recruited by early origins. 
Recent reports have indicated that the Forkhead transcription factors Fkh1 and Fkh2 are involved in early origin firing [11,17]. Although FKH1 and FKH2 are not required for origin licensing, the binding of Cdc45 to early origins in G1 seems to be Fkh1/2-dependent, and it has been suggested that the role of Fkh1/2 in origin firing would involve the establishment of replication timing domains and the spatial organization of origins [11]. Fkh1/2 binding sites are enriched in a subset of origins, but ChIP-chip analyses indicate that there is no strict correlation between the presence of consensus motifs and Fkh1/ 2 binding [20,21]. In addition, the presence of Fkh1/2 binding sites is not sufficient to confer early firing, and the number and distribution of Fkh motifs within the origin region seem to be important for conferring early origin activation [11,17], arguing for an additional role of the chromatin environment in the regulation of origin firing.

Chromatin modifications also play a role in the regulation of origin timing and several studies have demonstrated that increases in histone acetylation, either by deletion of the deacetylase $R P D 3$ or by artificial recruitment of the histone acetylase Gcn5, correlate with advanced origin firing $[9,10,12,22]$. However, the explanation as to how histone acetylation modifies replication timing has remained obscure.

Previous studies have shown that replication origins in budding yeast exhibit a specific nucleosomal configuration, consisting of a nucleosome-depleted region (NDR) next to the ACS element, flanked by well-positioned nucleosomes $[23,24]$. The correct organization of these origin-associated chromatin features is important for efficient replication initiation [25] and it would also be important for the timing of origin activation.

In light of the above, here we examined the contribution of the chromatin structure to the timing of origin firing by genome-wide analysis of the nucleosome organization at early and late origins and its relationship with epigenetic modifications, in particular with histone acetylation. Our findings show that early and late origins exhibit different nucleosomal patterns, which are influenced by the genomic context. We found that higher histone acetylation in rpd3 mutants was linked to local changes in chromatin structure, leading to a more open conformation at the affected replication origins. Finally, our analysis indicates that origin structure is dynamic during the cell cycle.

\section{Results}

\section{Early and late replication origins exhibit different nucleosome profiles}

To study the interplay between chromatin organization and replication timing, we generated high-resolution maps of nucleosome positions of the entire genome of $S$. cerevisae (see Methods). Previous experiments indicate that origin timing is established in early G1 phase [6] and we therefore determined the nucleosomal profile of replication origins from G1-synchronized cells (Figure 1A). Given the importance of aligning nucleosome maps relative to ACS to uncover chromatin structure in origins [23,24], we selected a subset of 317 origins with previously annotated ACS [26] (Additional file 1: Table S2). As shown in Figure 1A (top panel), the average nucleosome profile of replication origins is characterized by the presence of an NDR that covers the ACS element asymmetrically, flanked by well-positioned nucleosomes at both sides, as previously reported [23,24]. However, a diversity of chromatin structures in replication origins has been reported [23], and to identify variations in their nucleosomal patterns we performed a k-means clustering analysis to classify origins by their similarities in the NDR width and degree of occupancy of the flanking nucleosomes. Following those criteria, six origin groups with relatively distinct patterns of nucleosome distribution were chosen (see Methods) (Figure 1A, heat map). From classes 1 to 6 , the NDR width diminished from 193 to 129 bp as did the positioning and occupancy of the nucleosomes located at adjacent positions (Figures 1A and 1B).

Since replication origins displayed different types of nucleosomal configuration as well as a different timing of activation, we wondered whether early and late origins might be preferentially linked to some of the nucleosome profiles identified above. First, we independently aligned nucleosome maps corresponding to early and late origins groups relative to the ACS. The assignment of replication origins within early or late categories for our analysis was made according to timing data available from at least three independent studies (OriDB; [12,14,27-29] (Additional file 1: Table S2). Comparison of their average nucleosome profiles showed that early origins displayed a higher occupancy of nucleosomes immediately upstream $(-1)$ and downstream $(+1)$ from a broader NDR feature, along with a better positioning of the adjacent nucleosomes (Figure 1C).

Second, we examined the nucleosomal arrangement of early and late replication origins within the six types of nucleosome patterns described above. Although the 6 different classes of origin organization were found within early and late origin groups (Additional file 2: Figure S1A), quantification of the distribution of early and late origins between the different classes revealed a marked preferential distribution of early origins for classes 1 and 2 and of late origins for classes 5 and 6 (Figure 1D). Indeed, 60\% of early origins belonged to classes 1 and 2, while $65 \%$ of late origins belonged to classes 5 and 6 . Moreover, nearly $40 \%$ of early or late origins were clustered in class 1 or 6 respectively (asterisks in Figure 1D). The overlap between the profiles of classes 1 and 6, which include the highest proportion of early and late origins, shows a striking difference in chromatin architecture between them (Figure 1E).

Next, we wondered whether early and late origins, or their different chromatin structure, could be influenced by the genomic context in which origins are placed. In S. cerevisiae, 


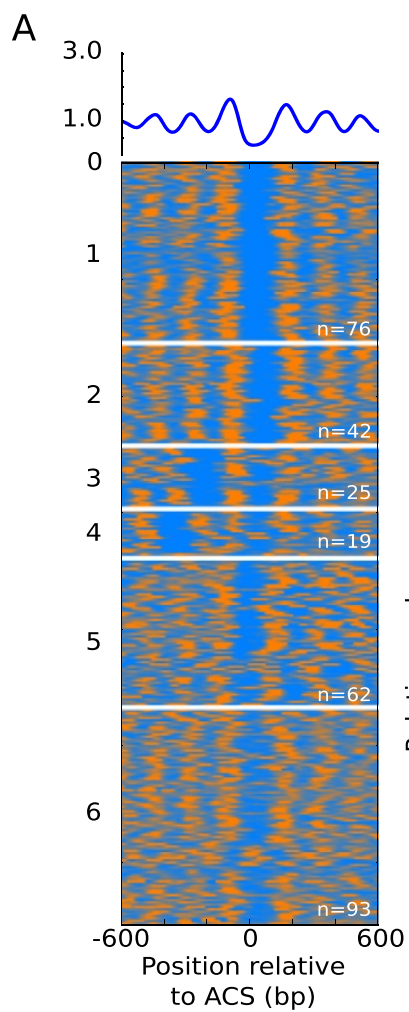

C

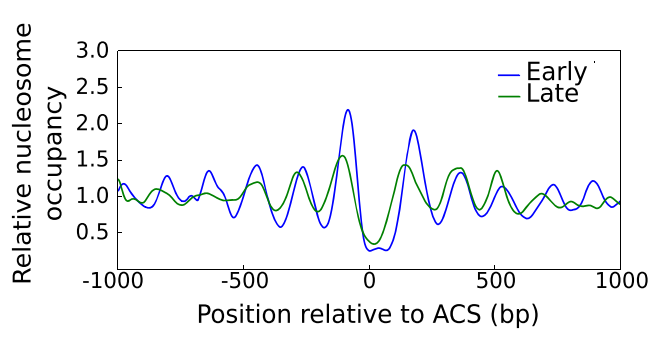

$\mathrm{E}$

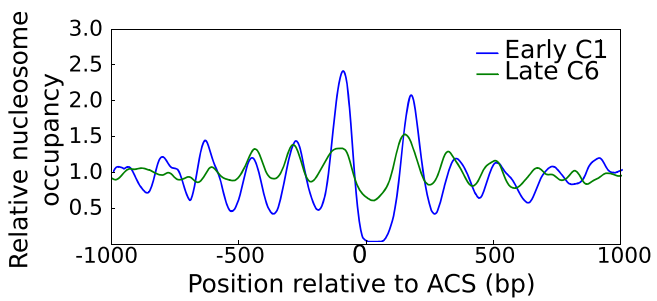

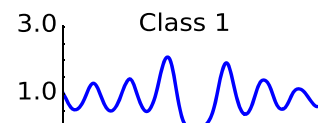

3.0. Class 2

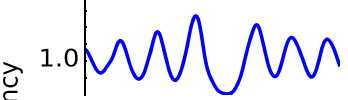

Class 3

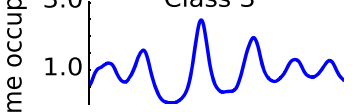

है 3.0 Class 4

บับ $1.0 \mathrm{Nm}$

$\sum_{\frac{1}{0}}^{D} 3.0$ Class 5

ฯ 1.0 N

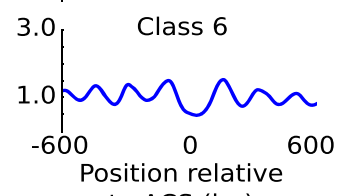

to ACS (bp)

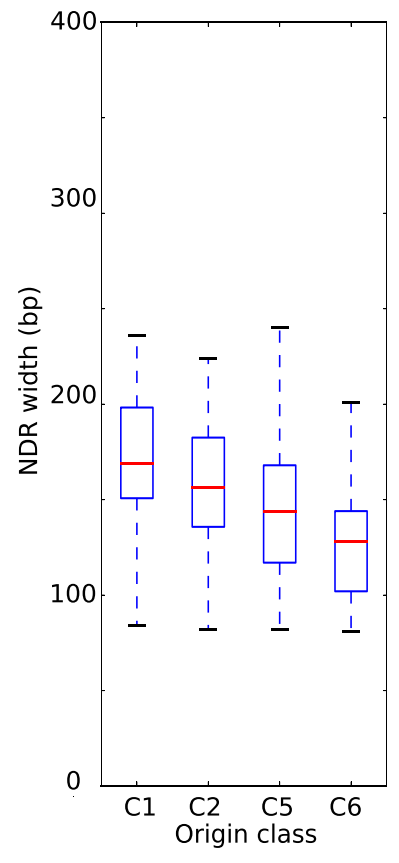

D
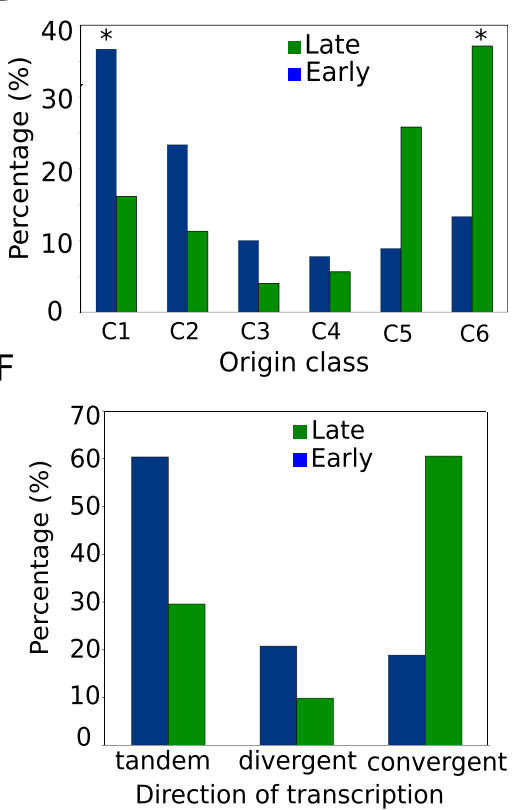

Figure 1 Early and late replication origins show different nucleosomal organization patterns. (A), Heatmap of nucleosome occupancy from 317 replication origins. ARSs are aligned on the $Y$ axis, and the distance from the ACS is indicated on the $X$ axis. Nucleosome occupancy is indicated in orange and nucleosome depletion is indicated in blue. Average nucleosome profiles for the six groups from the k-means clustering are plotted on the right (blue line). (B), Box plot analysis of the NDR width for origin classes shown in $\mathbf{A}$. Classes 3 and 4 were excluded due to their reduced sample size. The bottom and top of the box are the first and third quartiles, and the red band inside the box is the second quartile (the median). The ends of the whiskers represent the lowest and highest data still within $1.5 \mathrm{IQR}$ of the lower and upper quartiles respectively. (C), Comparative nucleosomal profiles of early (blue line) and late (green line) origin groups. (D), Percentage of early (blue) and late (green) replication origins included in the classes established in A. (E), Comparative nucleosomal profiles of class 1-early origins (blue line) and class 6-late origins (green line). (F), Percentage of early (blue) and late (green) replication origins located in intergenic regions between tandem, divergent and convergent transcripts. 
replication origins are mainly located at intergenic regions (IGR), which may be flanked by genes transcribed codirectionally, convergently or divergently. Replication origins in budding yeast appeared in these three types of IGR, but when we quantified the number of early and late origins located in each type of IGR, we found that they were not randomly distributed between them. In fact, while early origins can be found in all types of IGR with nearly the expected probability, late origins showed a strong bias for convergent IGR (Additional file 2: Figure S1B).

We asked whether this preferential localization would be related to their different chromatin architecture. To address this question we quantified the number of C1\&2-early and C5\&6-late origins located in each type of IGR, and we found that $60 \%$ of origins in each group mapped to IGR between tandem and convergent transcripts, respectively (Figure 1F). Moreover, class 6 was particularly enriched in convergent IGR, and the percentage of C6-late origins at this type of IGRs reached $71 \%$. Our results indicated that class 6 was linked to this type of gene organization (convergent transcription), and class 6 is highly enriched with late origins, therefore late origins are necessarily over-represented at convergent IGR. Origin-free NDR in convergent IGR were on average shorter and presented lower nucleosome occupancy than NDR located in IGR with gene units organized in tandem or divergently (Additional file 2: Figure $\mathrm{S} 1 \mathrm{C})$, resembling class 6-origin patterns. This might suggest that IGR with convergent transcription are prone to have lower nucleosome occupancy and shorter NDR.
In sum, although a nucleosome-depleted region next to the ACS element flanked by positioned nucleosomes is a common feature shared by all origins, the above analysis identified previously unknown separate modes of nucleosome organization in early and late replication origins. In addition, these early and late-nucleosomal patterns were preferentially located in different kinds of intergenic regions, which might condition their different chromatin structure and timing.

\section{Fkh-binding sites co-localize with the NDR in early origins}

Next, we investigated the relationship between factors involved in replication timing and chromatin organization. A role of Fkh1/2 transcription factors in early origin firing has been described [11,17]. However, although Fkh1/2-binding sites are widespread throughout the genome and are frequently found in origins, only Fkh1/2 consensus sites placed in very close proximity to ACSs confer early origin activation $[11,17]$. In fact, it has been recently reported that some early origins present two Fkh1/2-binding sites separated not less than $60 \mathrm{bp}$ and not more than $120 \mathrm{bp}$ [17], although the reason behind that is unknown. We argued that the distribution of Fkh1/2 motifs might be related to origin architecture.

To examine this possibility, we inspected the location of Fkh1/2-binding sites in replication origins in the context of chromatin organization. Thus, we searched for the Fkhbinding sequence throughout the genome and plotted the average enrichment of the Fkh motif in early or late origin clusters (red line) within the framework of their nucleosome profiles (blue line). Figure 2 shows that the Fkh-binding

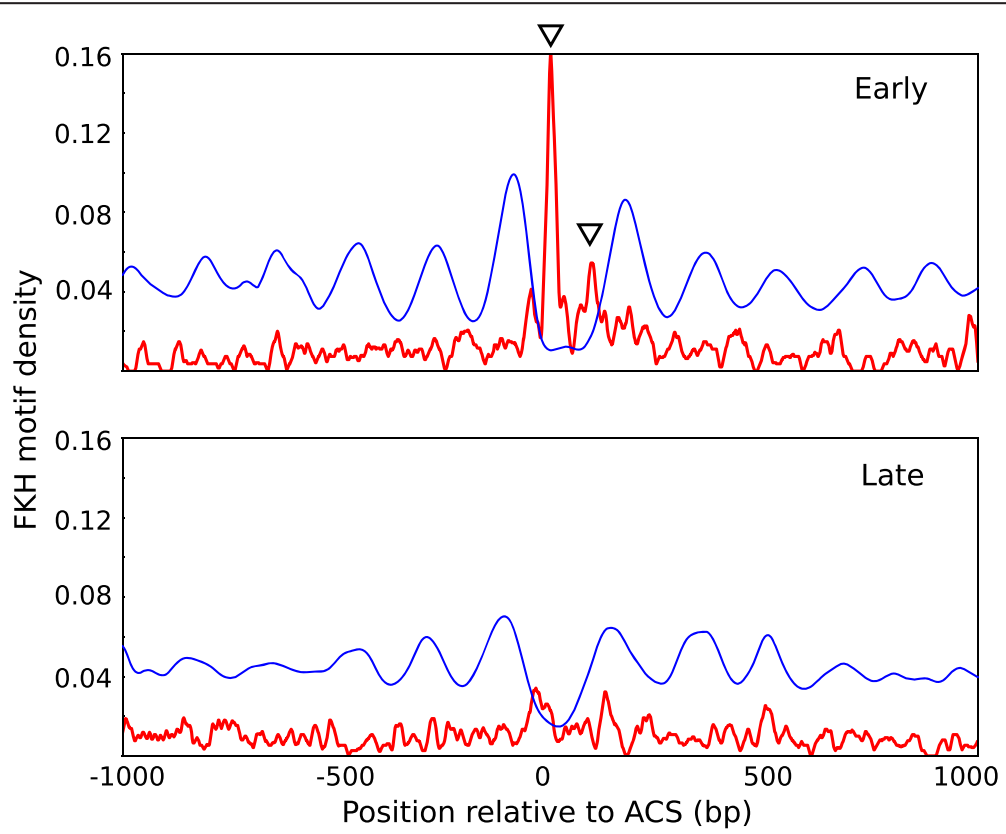

Figure 2 Fkh-binding sites are enriched in the NDR of early origins. Aggregated nucleosomal profiles (blue line) and Fkh-motif density (red line) were aligned relative to the ACS of early (top panel) and late (bottom panel) replication origins. 
sequence was highly represented only in early origins, as expected. In addition, our analysis indicated that there were two preferential Fkh-binding sites at early origins, and remarkably, both sites showed a precise co-localization with the NDR. These two Forkhead sites are located at each border of the NDR; the best represented is located in close proximity to ACS, while the other one is on average 80-100 bp apart, beyond a middle NDR region where Fkh sites are excluded.

These results advance our previous knowledge by showing that Fhk1/2 motifs are often positioned at the boundaries of the NDR, which could explain why critical Fkh1/2 motifs are restricted to the close vicinity of ACS and why they are not further apart than $120 \mathrm{bp}$. Therefore, the localization and distribution of Fkh1/2 motifs required for early origin function might be imposed or determined by the NDR element.

These data also suggest that the location of Fkh motifs within accessible chromatin is important for early origin firing. It would be possible that the higher accessibility of Fkh1/2 motifs located within the NDR may favour the binding of Fkh1/2 factors, or/and the interaction between Fkh and replication factors such as Cdc45 in early origins [11,17]. However, not all early origins contain Fkh-binding sites, and only $25.6 \%$ of the early origins considered in our analysis had this specific configuration of 2 Fkh sites close to the ACS, indicating that other factors must influence early firing too.
An increase in histone acetylation correlates with local nucleosomal remodelling in rpd3 mutants

Histone modifications also play a role in determining origin timing, and an increase in histone acetylation around origins induces earlier firing [9]. Our data revealed a correlation between origin structure and replication timing and we therefore wondered whether histone acetylation might modify replication timing by altering the chromatin architecture of replication origins. To examine this possibility, we generated genome-wide nucleosome maps in an rpd3 $\Delta$ mutant, where the RPD3 histone deacetylase gen had been deleted.

Despite Rpd3 playing a key role in the global deacetylation of the two core histones $\mathrm{H} 3$ and $\mathrm{H} 4$ [30], our maps showed that the general pattern of nucleosome distribution was mostly unaltered in the absence of Rpd3 (Figure 3 and Additional file 3: Figure S2). In fact, the average nucleosome organization of genes relative to the +1 nucleosome or relative to the Transcription Start Site (TSS) was very similar in the wild-type and rpd3A mutant strains (Additional file 3: Figure S2A). However, the analysis of individual genomic regions showed that although most nucleosomes occupied the same positions in both strains, some differences were observed at specific nucleosomes (arrows in Figure 3A and Additional file 3: Figure S2B), as in the case of the acid phosphatase PHO5 locus. Deletion of RPD3 increases the expression of the PHO5 gene and involves

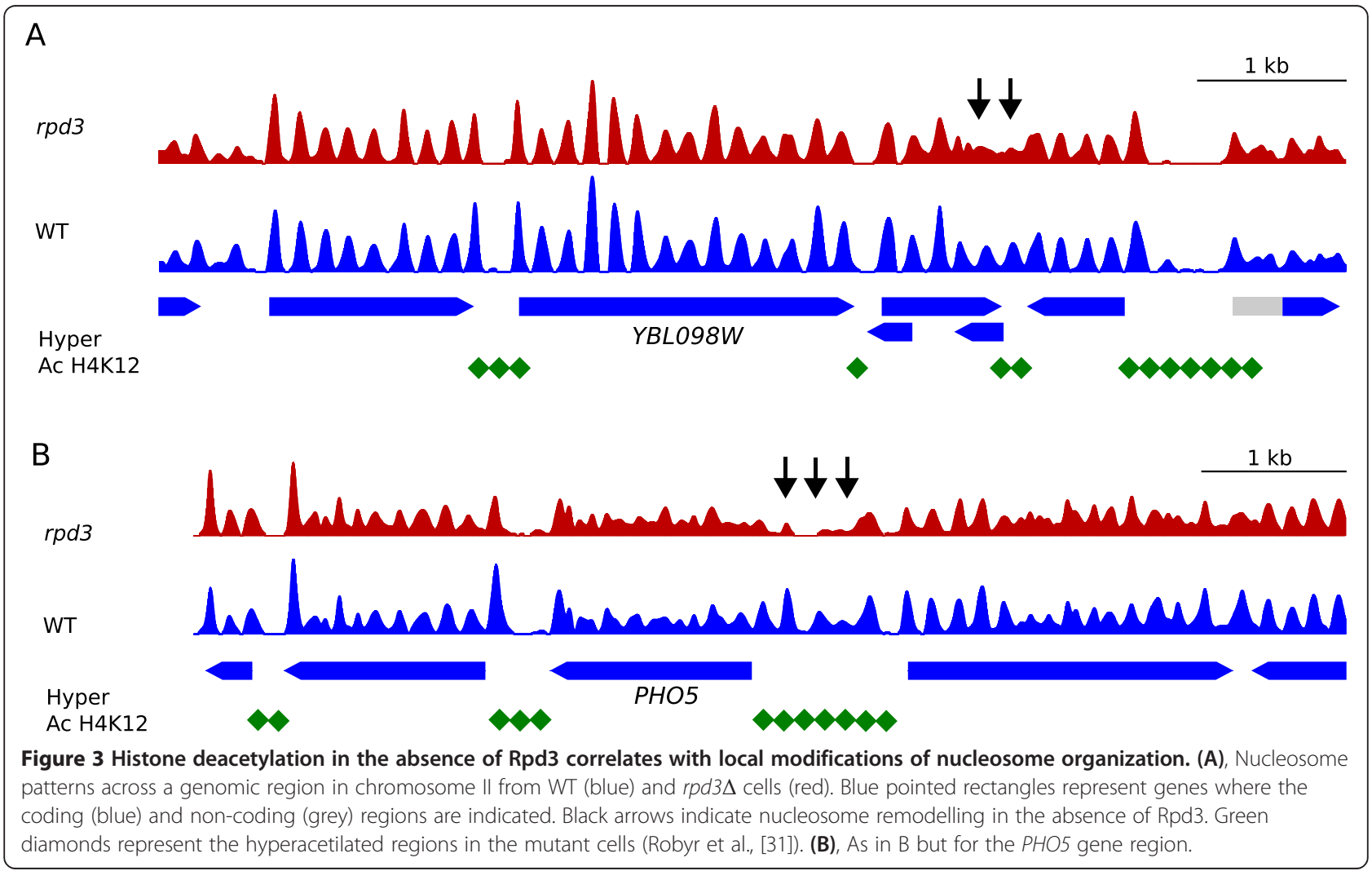


deacetylation along a $4.25-\mathrm{kb}$ region that includes PHO5 [30]. However, chromatin remodelling in the absence of Rpd3 was restricted to a few nucleosomes surrounding an NDR located upstream from the 5' end of the gene (Figure $3 \mathrm{~B}$ ). This observation indicated that histone deacetylation results in local modifications of nucleosome organization in key regulatory elements, which seem to have a great impact on transcriptional regulation, and perhaps also on replication timing.

\section{Deletion of the deacetylase RPD3 increases origin accessibility}

To explore the above possibility, we wondered whether chromatin structure in origin regions would also be modified locally in an Rpd3-dependent manner. Analysis of the average nucleosomal organization of replication origins from G1-synchronized cells showed that origins presented a NDR flanked by well-positioned nucleosomes in rpd3 $\Delta$ cells too and, also, that early and late origins displayed different nucleosome profiles when analyzed separately
(Additional file 4: Figure S3A). This result indicated that the different profiles observed at early and late origins were Rpd3-independent.

However, when we compared the nucleosome profile of replication origins between the wild-type and rpd3 $\Delta$ strains, we observed that the chromatin structure of origins was affected in the absence of Rpd3 in G1 (Figure 4A), but not in S-phase (Additional file 4: Figure S3B). Thus, rpd3 mutants had a wider NDR and lower occupancy of the -1 and +1 nucleosomes. Importantly, the average nucleosome pattern in NDR that did not colocalize with origins revealed no significant changes between the two strains (Figure 4B), indicating that the observed Rpd3dependent remodelling was origin-specific. To estimate the extent of the differences in the NDR length between wild-type and rpd3 $\Delta$ strains, we measured the width of the NDR of replication origins individually and plotted the average length for each strain. Figure $4 \mathrm{C}$ shows that the NDR size increased in rpd3 $\Delta$ mutants in origins, but not in the control group (see also Additional file 1: Table S2).

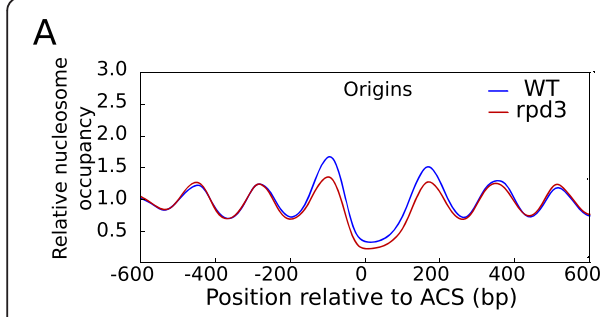

B

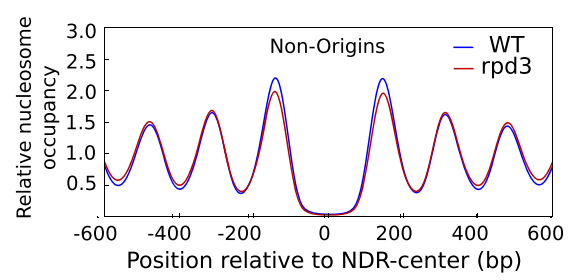

E

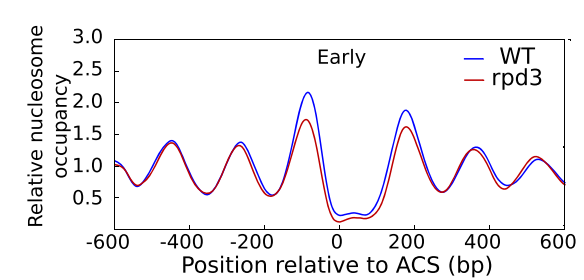

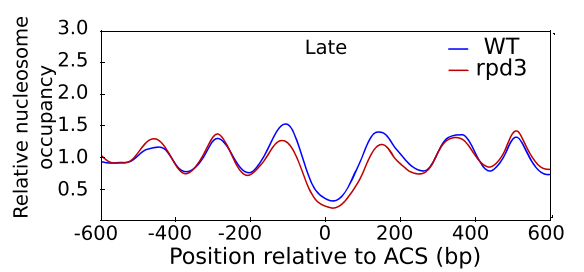

C

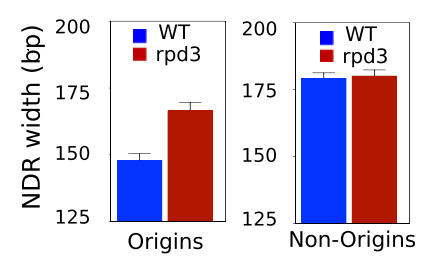

F

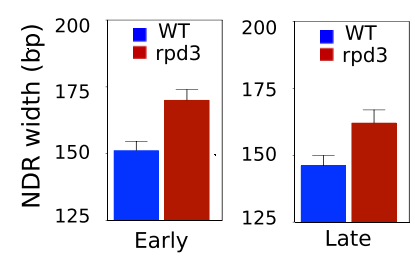

G
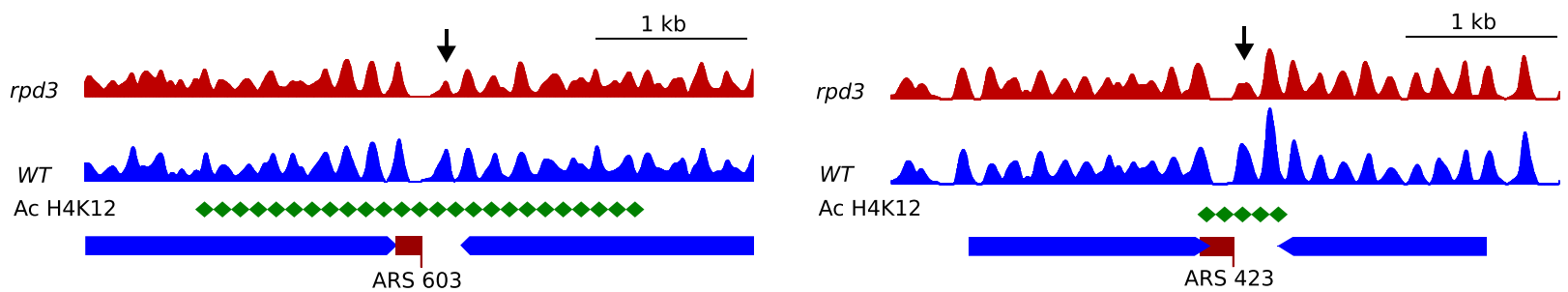

Figure 4 Origin accessibility is increased in the absence of Rpd3. Aggregated nucleosomal profiles of 318 NDR associated with replication origins (A) or 320 NDR non-associated with origins (B) from G1-arrested WT (blue) and rpd3A (red) cells were aligned relative to the ACS or to the NDR center, respectively. (C), Average NDR width of replication origins (left graph) or non-origin regions (right graph) from WT (blue) and rpd3 $\Delta$ mutants (red). (D), As in A but for early origins. (E), As in A but for late origins. (F), As in C but for early (left graph) and late origins (right graph). (G), Nucleosome patterns around the late origins ARS 603 and ARS 423 from WT (blue) and rpd3 $\Delta$ samples (red). Hyper-acetylated regions in the rpd3 mutant (Robyr et al., [31]; Vogelauer et al. [9]) are represented in green. Black arrows point to nucleosomes affected in the absence of Rpd3. 
To discriminate whether the G1-specific nucleosomal remodelling observed was affecting early or late replication origins or both, we analyzed the average nucleosome profiles of early and late origin groups independently. Figure 4D-E shows that chromatin structure was remodelled in both types of origins, although changes in the NDR width and occupancy in late origins were slightly more pronounced. According to this, the NDR size increased in rpd3 $3 \Delta$ mutants in both early and late origins (Figure 4F). Local modifications in the NDR region also included the aperture of an NDR that was occupied by a nucleosome in a wild-type strain. This NDR "opening" was taken place in the absence of Rpd3 in $21 \%$ of early and $39.2 \%$ of late origins with these characteristics, and an example is shown in Additional file 4: Figure S3D for ARS1410.

Chromatin remodelling in the de-acetylation mutant also affected the occupancy of nucleosomes flanking the NDR. These local modifications were examined in individual origin regions in the absence of $\operatorname{Rpd} 3$, and some origins such as ARS603 and ARS423 showed a noticeable decrease in occupancy in the +1 nucleosome (arrows in Figure 4G) while in others like ARS1413 the nucleosomes $-1,-2$ and -3 were also affected (Additional file 4: Figure S3E). When acetylation data were available $[9,31]$ for the origin analyzed, we found that the chromatinremodelled region was hyper-acetylated (Figure 4G).

Our data indicate that $\operatorname{Rpd} 3$ affects the nucleosomal configuration of many early and late origins. Similarly, Rpd3 affects the replication timing of numerous origins, including early and late ones $[9,12]$. We confirmed that origin replication timing was altered in our rpd3 $\Delta$ sample by examining origin activation of the early origin ARS305 and the late origin ARS603 during S phase as described in Additional file 5: Figure S4. Thus, 2D gel analysis indicated that when RPD3 was deleted the bubble arc for ARS305 was most prominent at $30 \mathrm{~min}$, whereas in the wild-type strain the maximum was observed at $45 \mathrm{~min}$ (Additional file 5: Figure S4A). In addition, we examined the position of the replication forks by monitoring the association of the Replication Protein A (RPA), a single-strand DNAbinding protein that has been shown to match the location of the replisome [32]. Chromatin immunoprecipitation (ChIP) experiments showed that the association of Rpa1 peaked at $5 \mathrm{~kb}$ away from ARS305 in rpd3 $\Delta$ cells while in wild-type cells Rpa1-association was restricted to the origin position (Additional file 5: Figure S4B). Since origin efficiency seems comparable in both strains, we concluded that ARS305 fires earlier in the mutant strain and therefore that more DNA will be synthesized before dNTP levels are depleted by HU. These data are in agreement with previous observations showing that the firing of ARS305 is advanced in rpd3 mutants [9]. When we examined the activation of ARS603 we detected initiation structures (Additional file 5: Figure S4A), indicating that in rpd3 $\Delta$ mutants late origins can escape checkpoint inhibition to a certain extent, as described [10]. However, the lower bubble arc signal in ARS603, suggested that late origin firing before critical dNTP depletion was taking place in a small proportion of cells. Therefore, replication timing was altered in rpd3 mutants, and so was the nucleosomal organization around origin regions.

Then we took the opposite approach, and we analyzed the nucleosome profile of a described set of origins (Additional file 1: Table S2) with advanced replication in the absence of Rpd3 [12]. Additional file 4: Figure S3C showed similar variations in the NDR-width and occupancy of flanking nucleosomes as described in Figures 4A-D-E. These results strongly suggest that nucleosome remodelling in rpd3 mutants is related with changes in replication timing.

Taken together, our results indicate that there is a correlation between changes in histone acetylation and replication timing with local modifications in origin architecture. Despite the fact that early and late specific chromatin patterns are conserved in rpd3 $\Delta$ mutants, the remodelling of key origin features such as the NDR and $-1 /+1$ nucleosomes leads to a more open conformation. Although it has been speculated that changes in histone acetylation can influence replication timing by modifying chromatin structure $[9,10]$, our results demonstrate for the first time that higher levels of histone acetylation caused by the deletion of the deacetylase RPD3 increase NDR opening.

\section{Origin architecture is dynamic during activation}

As mentioned in the Introduction, origin activation involves the modification of chromatin-bound replicative complexes and we wondered whether this process might entail modifications in origin structure during $S$ phase. To explore this possibility, we performed a genome-wide analysis of the dynamics of chromatin organization at replication origins before and after origin activation by comparing the nucleosomal pattern of synchronized populations in G1 and cells proceeding into S-phase in the presence of hydroxyurea (HU). The slow movement of replication forks in the presence of HU facilitates the analysis of replication dynamics and nucleosome organization during S-phase. Additionally, late origin firing is inhibited in the presence of $\mathrm{HU}$ by the replication checkpoint [33,34], providing a system to compare chromatin organization in active or inactive origins within the same replication cycle.

The analysis of nucleosome distribution in G1 and Sphase samples revealed that the majority of nucleosomes seem to occupy the same positions before and after replication initiation (see analysis of $8 \mathrm{~Kb}$ genomic regions at the ARS305 and ARS501 loci in Additional file 6: Figure S5). We noted that in early origin regions, 
and not in late ones, the average nucleosome signal was substantially higher during $S$ phase (Additional file 6: Figure S5A). When the differential between the nucleosomal signals from the S and G1 samples was represented (grey profile) a major S-phase signal coinciding with early origin regions was corroborated (Additional file 6: Figure S5B). Higher nucleosome signals would be expected at those regions due to an increase in DNA copy number after replication from early origins. In fact, analysis of the ARS305 region indicated that a major SG1 differential signal extended for 2-2.5 kilobases on either side of the origin (Additional file 6: Figure S5B), coinciding with the previously described length of replication intermediates accumulated at HU-blocked replication forks [33,35]. Indeed, when nucleosome signals were corrected for copy number (see Methods), the G1 and S-phase signals in early origin positions became similar, while late origins regions remained unchanged (Additional file 6: Figure S5C). Therefore, the location and extent of a region with elevated nucleosome signals in S-phase relative to G1 mark out origin activation and newly replicated chromatin, and hence nucleosome mapping by deep sequencing is a suitable approach for analyzing both chromatin and replication dynamics at genomic scale.

However, a careful comparison of the average nucleosome configuration in replication origins regions revealed that origin structure changed from G1 to S phase, and remarkably, different changes were observed in early and late origin groups (Figure 5 ). In early origins, $+1,+2$ and +3 nucleosomes were shifted to the left in S-phase cells (green line) compared to G1 cells (blue line) (Figure 5A). By contrast, in late origins, the NDR print was wider in the S-phase sample and, in this case, a clear reduction in occupancy of the -1 and +1 nucleosomes was also observed (Figure 5B).

This nucleosome rearrangement was also detected in individual examples such as the early origins, ARS305 and ARS731, and late origins, ARS603 and ARS501 (Figure 5C and 5D). The high resolution achieved in our nucleosome maps was able to detect modifications at individual nucleosome positions and, in fact, early origins showed a clear displacement of the first nucleosomes opposite to the ACS towards the inner part of the NDR during Sphase (Figure $5 \mathrm{C}$ ), while the rest were maintained at the same positions as in G1. By contrast, although changes in nucleosome positions were also restricted to the vicinity of the ACS in late origins, nucleosome displacement was directed outside the NDR and the occupancy of +1 nucleosome was drastically reduced in late origins such as ARS603 (Figure 5D).

The above observations revealed that the structure of the NDR is dynamic during replication but we then wondered why NDR-modulation would be different at early and late origins during $\mathrm{S}$ phase. We reasoned that the observed changes might be linked to origin activation, which in HU should take place exclusively at early origins. To confirm the unique activation of early origins in our experimental conditions, we performed 2D gel analysis of DNA replication intermediates on ARS305 and ARS603 as previously described [36]. In ARS305, the replication bubble arc indicated origin activation (Figure 5E). By contrast, when the membranes were reprobed to analyze ARS603 activation, no initiation structures at this late origin were observed. Therefore, one possibility was that the NDR-conformation would be different in origins before and after origin firing (early origin $=$ post-firing state or late origin $=$ pre-firing state in $\mathrm{S}$ phase in the presence of $\mathrm{HU}$ ).

One prediction of this hypothesis is that the NDR would be modulated at replication origins at their time of origin firing. To test this possibility, we compared the nucleosome organization of the ARS603 region before and after origin activation during an unperturbed $S$ phase (Figure 6 and Additional file 7: Figure S6) by micrococcal nuclease (MNase) analysis. In this experiment, G1-synchronized cells were released into $\mathrm{S}$ phase, and different samples were taken from 20 to 80 minutes (Additional file 7: Figure S6). In each sample, chromatin was digested with increasing amounts of MNase, followed by digestion with an appropriate restriction enzyme and Southern hybridization with a terminal probe. The results shown in Figure 6 indicated that the NDR region colocalized with two MNasehypersensitive sites flanking a protected middle region and, importantly, the lower MNase site was reduced in intensity (asterisk) while the upper MNase-hypersensitive zone was expanded (arrow) from 40 minutes onwards, coinciding with the activation time of late origins (Additional file 7: Figure S6B).

We conclude that origin nucleosomal configuration is dynamic during the cell cycle and propose that origin activation is associated with local changes in chromatin organization. Changes in chromatin architecture at origins seem to be intimately linked to their timing of activation and thus chromatin modulation in early and late origins is separated temporally. It is possible that changes in chromatin organization in origins might be required to allow the correct assembly/disassembly of replication factors before and after origin activation.

\section{Discussion}

To elucidate the role of chromatin structure in the timing of origin firing in budding yeast, we conducted a high-resolution analysis of genome-wide nucleosomal organization at origins. Our results revealed distinctive nucleosomal configurations in early and late replication 


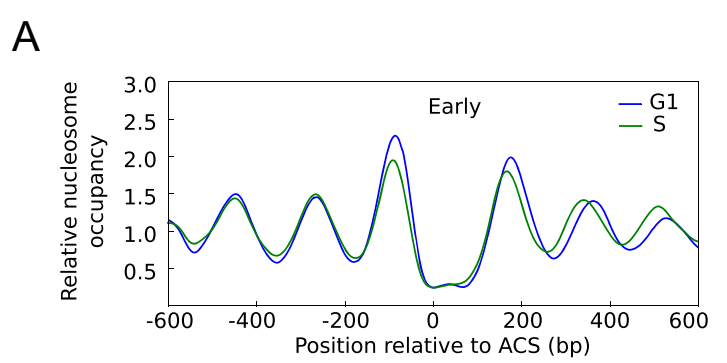

C

ARS 305

G1
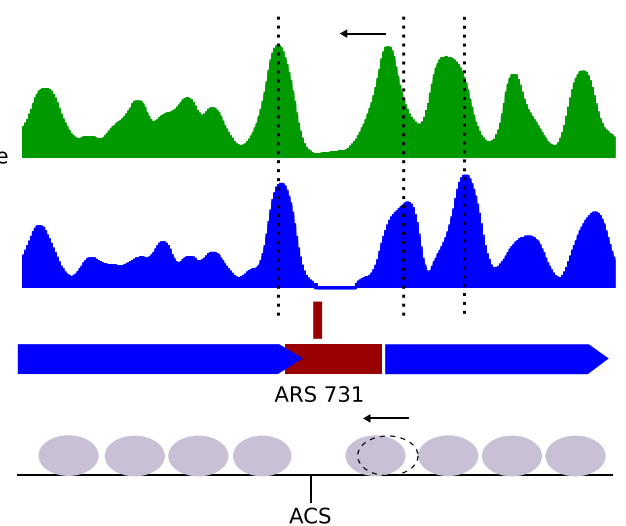

B

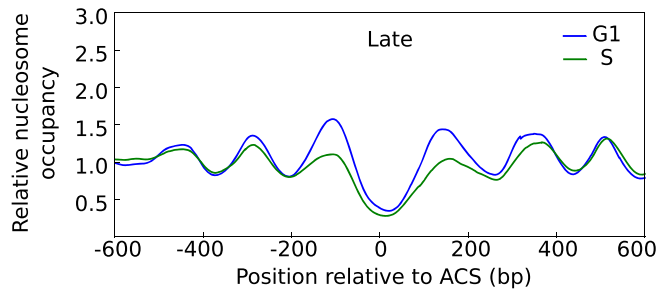

D

$200 \mathrm{bp}$
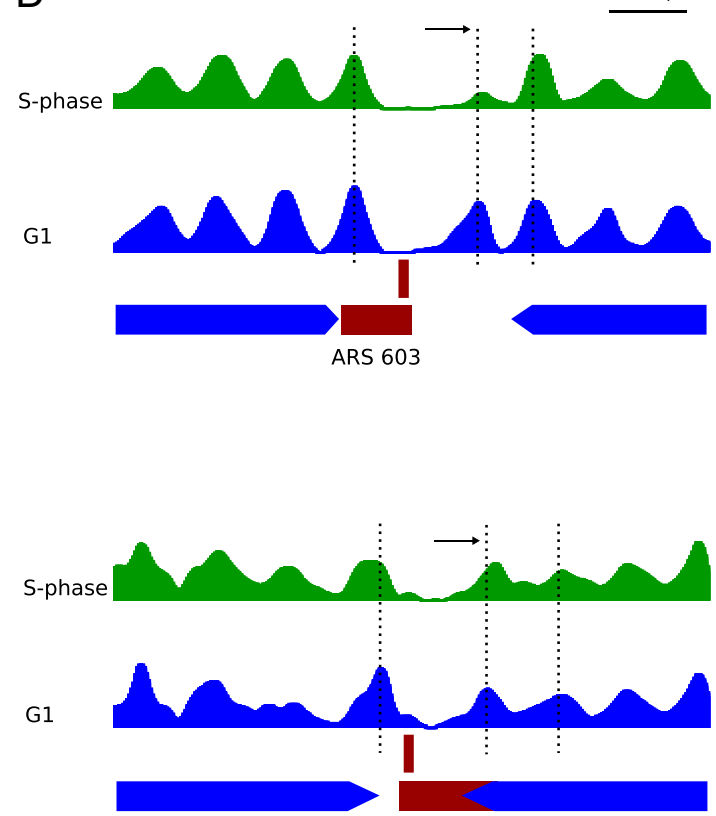

ARS 501

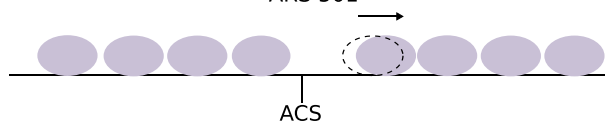

E

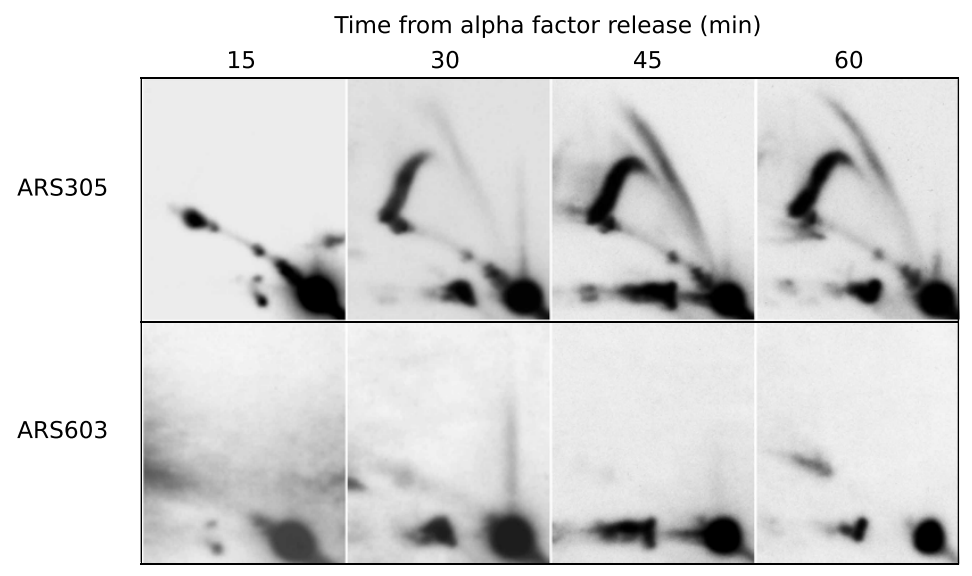


(See figure on previous page.)

Figure 5 Origin architecture is remodelled during activation. Average nucleosomal profiles from early (A) and late (B) origin groups in G1 (blue line) and S-phase (green line). (C), Nucleosome patterns of the early origins ARS 305 (top panel) and ARS 731 (bottom panel) during G1 (blue) and S-phase (green). Genes, replication origins and ACS are represented as in Figure 3. Dotted lines indicate the center of the nucleosomes flanking the origins during G1. Nucleosome remodelling at early origins is summarized in a schematic diagram where grey ovals represent nucleosomes in S-phase and the dotted nucleosome indicates the position of the $+1 \mathrm{~N}$ (the first nucleosome downstream of the ACS) in G1. Nucleosome displacement is represented by a black arrow. (D), As in C but for the late origins ARS 603 and ARS 501. (E), Neutral/neutral 2D gel analysis was performed to analyze ARS305 and ARS603 activity. Genomic DNA was prepared from cells released from a-factor arrest into YPD + 0.2 M HU and collected at 15, 30, 45 and 60 min.

origins. The high resolution of our nucleosome maps, together with the independent analysis of early and late origins clusters were critical to disclose these different nucleosomal architectures, emphasizing the notion that the average nucleosome profile of all replication origins hides important variations in origin structure.

Previous studies have suggested that genomic regions upstream from genes tend to replicate early, while regions containing a gene end tend to replicate late [23]. In agreement with this, our analysis indicates that there is a strong coincidence of the major late patterns $(5 \& 6)$ at convergent IGR that contain two gene ends, and moreover, they seem to be almost completely excluded from regions that do not contain any gene end (divergent IGR). The situation seems to vary further for early origin patterns, because these can be found in regions that coincide with transcription initiation or termination, or both. However, it is unclear why late origin patterns are highly represented at convergent IGR. It is possible that a higher rate of transcription termination at convergent IGR may interfere with nucleosome stability, leading to the lower occupancy observed in late origin patterns. This possibility is supported by the fact that other convergent IGR, non-associated with replication origins, also presented low nucleosome occupancy.

Alternatively, the co-localization of origins and transcription initiation sites (TSS) may promote early firing. In this sense, replication origins from several organisms

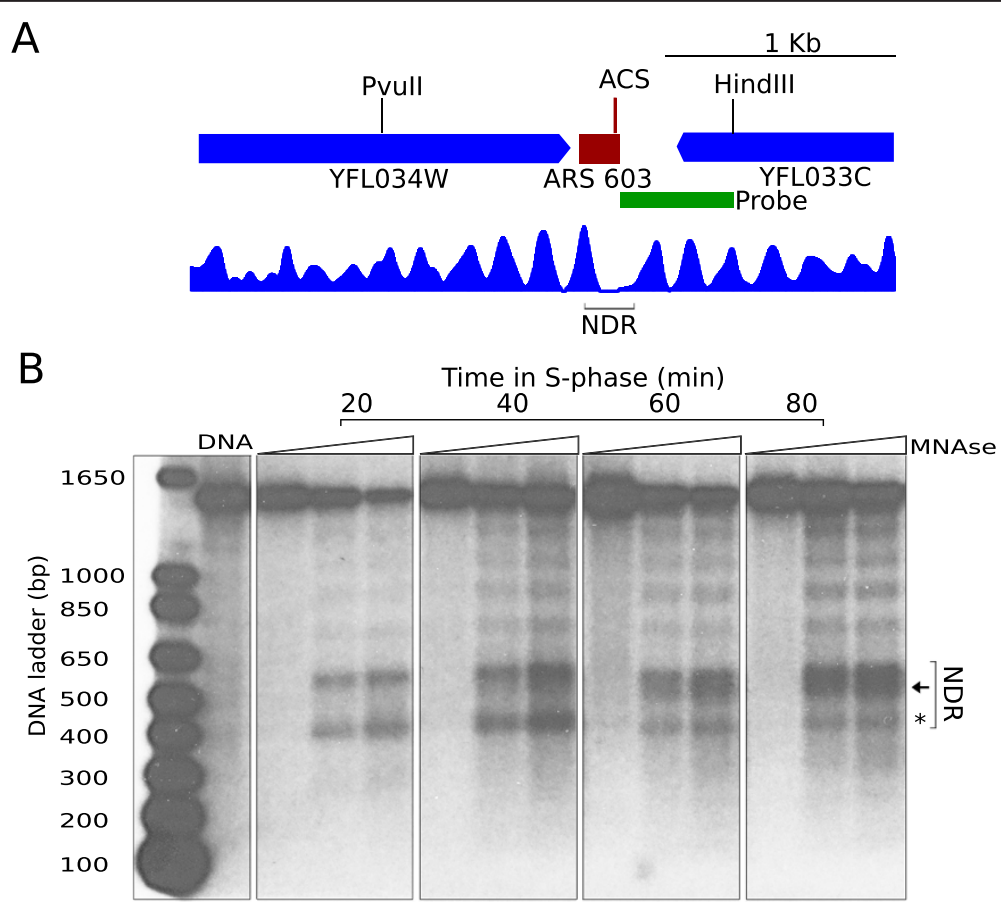

Figure 6 The NDR is modified along S phase. (A), ARS603 is represented by a red rectangle and flanking genes, YFL034W and YFL033C, by blue ones. Vertical bars indicate sites for digestion with restriction enzymes Pvull and Hindlll. Green rectangle represents the end-terminal probe. The nucleosomal profile from the G1 sample is presented below. (B), Naked DNA and chromatin from wild-type cells collected at 20, 4060 and 80 min after a-factor arrest and release into YPD were digested with increasing amounts of MNase (indicated with triangles) and subsequently digested with Pvull and HindIII. Samples were electrophoresed, transferred onto a membrane and hybridized. A ladder of polynucleosomes is observed with a prominent region of hypersensitivity to MNase corresponding to the NDR (indicated in brackets in A, B). The black arrow and asterisk point to the changes in chromatin organization observed in the NDR at later time points. 
frequently contain transcription factor-binding sites [37], arguing for a role of TF in origin regulation. In S. cerevisiae, the transcription factor Abf1 seems to help origin activation [38], and importantly, the transcription factor Fkh is involved in early origin firing $[11,17]$. Therefore, it is possible that origins located in the vicinity of a promoter would be more likely to bind Fkh1/2 transcription factors, which can strongly influence the selection of these origins for early firing. Supporting this possibility, $81 \%$ of early origins enriched with Fkh1/2-binding sites were in IGRs containing at least one promoter, while only $19 \%$ were in promoter-free regions. However, only $25.6 \%$ of the early origins analyzed contain Fkh-binding sites, suggesting that the role of TF in origin activation might not be exclusive to Fkh and that other TFs could also contribute to early firing in other origins. In fact, binding sites for other transcription factors have been found close to replication origins and have been implicated in origin activation in both yeast and mammals [39-42]. Future examination of the interactions between transcription and replication factors should provide important information about this issue.

Regarding the distribution of Fkh-binding motifs, the overlapping of predominant sites with the NDR origin feature suggests that the location of Forkhead sites within an open/accessible chromatin region is important for origin regulation. In addition, the particular localization and spacing of Fkh sites within NDR raise the possibility that the binding of TFs such as Fkh1/2 might also contribute to the maintenance of a nucleosome-free region and to the positioning of proximal nucleosomes, similar to the previously described role of ORC-binding [25].

The fact that both ORC and Fkh1/2-binding sites are restricted to the NDR suggests that the NDR is critical for accommodating functional complexes in the origins. The average NDR size from origin patterns 1-6 varies from 193 to $129 \mathrm{bp}$, indicating that different NDR widths are valid for origin activity. However, classes 1 and 2, with the broadest NDRs, and classes 5 and 6, with the narrowest NDRs, generally correlate with early and late replication respectively, indicating that NDR width might influence the timing of origin replication.

We propose that the different affinities of initiation factors for early and late origins would be determined by their different origin structure. Thus, initiation factors might have a higher affinity for early origins that generally adopt class 1 and 2 conformations and show broader and better defined NDRs. The importance of an appropriate NDR width in origin timing is highlighted by the observation that previously described dormant origins such as ARS301, ARS302, ARS303 and ARS320 show either a closed NDR or an extremely small NDR (97 bp) (Additional file 8: Figure S7). Dormant origins bind a pre-RC and are licensed in G1 [4], and if given enough time they are competent to initiate replication $[43,44]$. This small NDR size probably imposes a critically low affinity for regulatory factors in these origins, being excluded for firing in a normal replication cycle. The higher occupancy by $-1 /+1$ nucleosomes in early origins suggests that the interaction of the initiation factors with the origin region or with some components of the pre$\mathrm{RC}$ complex might be facilitated or mediated by interaction with these nucleosomes. In fact, previous studies have reported an interaction between transcriptional regulators and +1 and +2 nucleosomes $[45,46]$.

Our results also revealed that origin architecture was actively modulated during origin firing. To visualize replicationdependent origin remodelling it is important to compare high-resolution maps from synchronized G1 and S samples, perhaps explaining why it passed unnoticed in previous studies performed on asynchronous cultures and/or with tiling microarrays [23,24]. An interesting possibility is that origin structure might be influenced by the different replication complexes or factors bound to the origin before and after origin firing. This is in agreement with the fact that some pre-RC components and/or initiation factors are bound and released from chromatin before and after origin activation $[2,3]$. Interestingly, some initiation factors such as Sld3 and Cdc45 can be detected specifically at early firing origins during G1 [11,18,19]. These essential initiation factors are present in the cell in limiting amounts $[15,16]$, and it has been suggested that they are recycled from early to late origins during $S$ phase. Therefore, the dynamic of nucleosomes and replication proteins during the process of origin activation might be related, and we are currently examining this possibility.

In addition, we also examined the contribution of histone acetylation to early origin firing at the level of chromatin organization. Our data indicate that increased levels of histone acetylation around origins induce local modifications in chromatin organization, creating a more accessible structure. One possibility is that histone acetylation might weaken histone/DNA interactions, diminishing the chromatin association of ACS-flanking nucleosomes and altering NDR formation. The resulting open conformation in origins might facilitate, either directly or indirectly (through interaction with other chromatin-bound factors), the recruitment of critical replication factors to origins.

Taken together, the results reported argue for an important contribution of the chromatin structure to origin selection and firing, and help to understand the interplay between replication regulators such as Fkh1/2, epigenetic modifications, and chromatin structure.

\section{Conclusions}

Coordinated origin firing is important to ensure complete replication of the entire genome and recent evidences suggest that the replication temporal program can influence 
genome stability $[47,48]$. In this work, we have analyzed the role of chromatin structure in replication timing at the level of nucleosome organization. Our data highlight the importance of chromatin features, such as the NDR and nucleosomes +1 and -1 , in the regulation of origin activation and timing. Thus, early origins present broader NDR features and a high occupancy of flanking nucleosomes while tighter NDR and a lower occupancy appear in late origins. Besides, factors involved in the establishment of origin timing such as histone deacetylation induce local modifications in these key origin features, leading to a more open origin conformation. Similarly, Fkh transcription factors seem to be in close relationship with origin structure, as suggested by the overlap of predominant Fkh-binding sites with the NDR origin feature.

Origin activation is also associated with local changes in chromatin organization. Although the specific chromatin conformations of origins are already established in G1, origin structure is dynamic during origin activation. It has been described that chromatin structure affects origin function [25], and it would be possible that origin architecture can also affect the affinity and efficiency of the formation of replication complexes and, therefore, influence the timing of origin firing.

\section{Methods}

\section{Yeast strains and growth conditions}

The strains used in this study are described in Additional file 9: Table S1 and derived from W303-1a (MATa ade2-1 ura3-1 his3-11 15 trp1-1 leu2-3 112 can1-100). Yeast cultures were grown in YP medium (1\% yeast extract, $2 \%$ bacto peptone) supplemented with $2 \%$ glucose (YPD) at $23^{\circ} \mathrm{C}$ to a final concentration of $0.7 \times 10^{7}$ cells $/ \mathrm{ml}$. Cells were synchronized in G1 phase by adding $5 \mu \mathrm{g} / \mathrm{ml} \alpha$ factor $(\alpha \mathrm{F})$ for one generation time and released into fresh medium in the presence/absence of $0.2 \mathrm{M}$ hydroxyurea (HU) depending on the experiment.

\section{Preparation of mononucleosomal DNA}

Previously published nucleosome maps were mostly obtained from asynchronous cultures, which would make difficult to detect nucleosome dynamics during different stages of the cell cycle. To overcome this limitation, we generated nucleosome maps from G1 and S-phase synchronized samples. Thus, $2 \times 10^{9}$ cells were collected at $\mathrm{G} 1$ and at 60 minutes in S-phase in the presence of $0.2 \mathrm{M}$ HU for preparation of mononucleosomal DNA and for ChIP experiments (see below). Preparation of mononucleosomal DNA was adapted from [49]. Spheroplasts were induced by treatment with $10 \mathrm{mg}$ of zymolyase $20 \mathrm{~T}$ for $10 \mathrm{~min}$ at $30^{\circ} \mathrm{C}$, and mononucleosomal fragments were generated by digesting DNA with 600 or 450 units/ml (WT and rpd3 strains respectively) of micrococcal nuclease (MNAse) at $37^{\circ} \mathrm{C}$ during 10 minutes. The amount of
MNase was optimized experimentally for each strain to generate an 80:20 ratio of mononucleosomes to dinucleosomes, as described [50].

\section{Sequencing and nucleosome maps representation}

Mononucleosomal DNA was sequenced using the singleend sequencing protocol in an Illumina Genome Analyzer IIx. All sequencing data are deposited in the Gene Expression Omnibus (GEO) database under the accession number [GSE54377]. 23117530 to 28511688 single reads 40 nucleotides long were aligned with the reference genome of S. cerevisiae S288C (R64-1-1 assembly 3/2/2011). For the alignment we used Bowtie with no mismatches allowed. and the average genome coverage obtained was from 76 to 95 -fold. This high level of resolution is a critical factor to detect subtle local modifications at individual nucleosome positions. The sequencing coverage for every nucleotide was normalized by the average genomic coverage. The nucleosome positioning profile was generated as described [51]. Briefly, the average spacing between boundary peaks for individual nucleosomes was calculated from the smoothed signal generated with Python "Pywavelets" (with the multilevel 1-D biorthogonal wavelet decomposition/reconstruction tool). Then, the midpoint position of each nucleosome was defined and the resulting combined profile was wavelet-smoothed. For the copy number correction shown in Additional file 6: Figure S5, the ratio of mapped reads from the G1 and S-phase nucleosomal samples to the naked-DNA samples from the same time points (SRR398641 and SRR398652 files deposited in SRA database) was calculated. The number of reads from the G1 and S naked-DNA samples were corrected to obtain an average number value across the genome of one, as previously described in replication timing profiles analysis [52]. The resulting signal was then smoothed with a $1000 \mathrm{bp}$ window, step $1 \mathrm{bp}$.

Clustering, analysis of NDRs, TSS and search for Fkh motif A list of replication origins with annotated ACS in the $+/-20$ nt limits of each origin was obtained from OriDB and those origins that mapped on a repetitive region were excluded. To generate the average nucleosome profiles, the nucleosome maps were aligned relative to the ACS and were oriented with respect to the T-rich strand. The nucleosome profiles of the $[-600,600] \mathrm{nt}$, centered on each ACS sequence, from the WT G1 sample were clustered using a k-mean clustering function with 10.000 iterations. We used 4 to 8 groups in our analysis, and six groups were finally chosen because they accomplished the best consensus between cluster size and information. Nucleosome-depleted regions (NDRs) were defined as regions spanning at least 80 nucleotides as described [53], with a normalized sequence coverage lower than 0.4 . The annotations of [54] were used as a TSS reference. The whole genome 
was searched for the FKH motif (RTAAAYA) previously described [17] by using the tool FIMO with a maximum threshold of 0.001 . We cured it by deleting all non-strict coincidences with the motif.

\section{Two-dimensional gel analysis}

DNA was isolated from $2 \times 10^{9}$ cells using a G-20 column (QIAGEN) according to the manufacturer's instructions. DNA fragments digested using XbaI and EcoRI (Fermentas) or BamHI and NcoI (Fermentas) were separated by neutral/neutral two-dimensional agarose gel electrophoresis as described [55].

\section{Chromatin Immunoprecipitation (ChIP) and quantitative real-time PCR (qPCR)}

ChIP extracts were prepared from $7.5 \times 10^{8}$ cells as described [56]. Immunoprecipitation was carried out overnight with $5 \mathrm{mg}$ of anti-PK antibody (SV5-Pk1 Fisher Scientific Pierce) coupled to magnetic beads (Dynabeads, Invitrogen). Uncoupled Dynabeads were used for background controls. The DNA fragments recovered from the whole-cell extract and ChIP samples were quantified by real-time PCR using the BioRad-CFX96 system. The qPCR signals obtained from the ChIP samples were normalized by the signals obtained from an input sample (percent IP). Primer sequences and amplification reactions are indicated in Additional file 9: Table S1. Primers were tested to achieve amplification curves with a slope of $3.3 \pm 10 \%$ and $\mathrm{R} 2$ value $>0.99$. All real-time PCRs were performed in duplicate with SYBR Premix Ex Taq (TaKaRa) in two independent experiments.

\section{Digestion with MNase and indirect end-labelling analyses} $2 \times 10^{9}$ cells were permeabilized as described above and spheroplasts were split into six fractions in NP-buffer and treated with increasing amounts of MNase (Fermentas) (0, 0.5, 1, 1.5, 2 and 3 units $/ \mathrm{ml}$ ) for 10 minutes at $37^{\circ} \mathrm{C}$. DNA was purified and quantified. For each sample, $2.5 \mu \mathrm{g}$ of DNA were digested with HindIII and PvuII enzymes (Fermentas), separated by electrophoresis in a $1.5 \%$ agarose gel, blotted and hybridized to an specific end-terminal probe.

\section{Availability of supporting data}

The data sets supporting the results of this article are available in the [GSE54377] repository, [http://www.ncbi. nlm.nih.gov/geo/query/acc.cgi?acc=GSE54377].

\section{Additional files}

Additional file 1: Table S2. Origin data set.

Additional file 2: Figure S1. Early and late replication origins are preferentially located in different IGR. (A), Average nucleosome profiles of early (blue line) and late (green line) replication origins for each of the six groups established by the k-means clustering shown in Figure 1A. (B),
Frequencies of expected (grey) and observed early (blue) and late (green) replication origins in IGR between tandem, divergent and convergent transcripts. Frequencies of IGR between tandem, divergent and convergent transcripts are 50\%, 25\% and 25\% respectively. (C), Average nucleosomal profiles from NDR in tandem, divergent and convergent IGR that are non associated with replication origins.

Additional file 3: Figure S2. The nucleosomal landscape in S. cerevisiae is largely maintained in the absence of Rpd3. (A), Aggregated nucleosomal profiles of WT and rpd $3 \Delta$ mutants from G1 (blue and red lines, respectively) and S-phase cells (green and yellow lines) were aligned to the midpoint position of the +1 nucleosome $(+1 \mathrm{~N})$, as described in Soriano et al. [51], and to the transcription start site (TSS). The coordinates of TSS have been reported by Lee et al. [54]. (B), Nucleosome patterns across a chromosome VII region from WT (blue) and $r p d 3 \Delta$ cells (red) as in Figure 3.

Additional file 4: Figure S3. Analysis of nucleosomal profiles in early and late replication origins in the absence of Rpd3. (A), As in Figure 1C but for rpd $3 \Delta$ cells. (B), Comparative nucleosomal profiles from WT and rpd $3 \Delta$ cells in G1 (left panel) and S-phase (right panel). (C), Aggregated nucleosomal profiles of 51 Rpd3-regulated origins described in Knott et al., [12] from WT (blue) and rpd3 $\Delta$ (red) cells were aligned relative to the ACS. (D), (E), Nucleosome patterns around the origins ARS 1410 and ARS 1413 from WT (blue) and rpd3 $\Delta$ samples (red). Black arrows point to nucleosomes affected in the absence of Rpd3.

Additional file 5: Figure S4. Replication dynamics in HU-treated $r p d 3 \Delta$ cells. (A), Analysis of origin activation by two-dimensional gel electrophoresis of ARS305 and ARS603 origins in wild-type and rpd3 3 cells. Genomic DNA was prepared from cells released from a-factor arrest into YPD + 0.2 M HU and collected at 15, 30, 45 and $60 \mathrm{~min}$. Black arrows indicate the sample with maximum intensity of the bubble arc in ARS305 (top panels). Arrows point to bubble arcs in ARS603 (bottom panels). (B) ChIP analysis of Rfa1-PK was performed in wild-type (left graph) and rpd3 $\Delta$ cells (right graph). Cells with PK-tagged Rfa1 were synchronized in G1 and released into rich medium containing $0.2 \mathrm{M} \mathrm{HU}$ for $60 \mathrm{~min}$. ChIP was performed with a-PK antibody. Histograms represent the percentage of immunoprecipitated DNA relative to the input. The PCR primers pairs correspond to the ACS and adjacent regions at the early origin ARS 305. Standard deviation bars are indicated.

Additional file 6: Figure S5. Higher nucleosome signals correlate with origin activation. (A), Average nucleosomal profiles from early and late origin groups in G1 (blue line) and S-phase (green line). (B), Nucleosome patterns across 8 kilobases of the $S$. cerevisiae genome encompassing the early origin ARS 305 (top) and the late origin ARS501 (bottom) from G1 (blue) and S-phase (green) cells. The differential signal between S-phase and G1 data is shown in grey. Genes, replication origins and ACS are represented as in Figure 3. (C), As in A, but nucleosome signals were corrected for copy number as described in Methods.

Additional file 7: Figure S6. Nucleosome and replication analysis during an unperturbed S-phase. Wild-type cells with PK-tagged Rfa1 were synchronized in G1 and released into rich medium. Samples were collected at the indicated time points for flow cytometric, ChIP and MNase analysis. (A), DNA content was measured by flow cytometry (B), Immunoprecipitated DNA was analyzed for the presence of ARS305

(circles), ARS609 (squares) and ARS501 (triangles) sequences by qPCR as described above. (C), Complete gel corresponding to Figure 6B, where only the three first lanes were shown for each time point.

Additional file 8: Figure S7. Dormant origins present closed or small NDR. Nucleosome patterns across a chromosome III region including ARS301, ARS302, ARS303 and ARS320. Genes, replication origins and ACS are represented as in Figure 3.

Additional file 9: Table S1. Yeast strains and primer sequences.

\section{Competing interests}

The authors declare that they have no competing interests.

\section{Authors' contributions}

IS and ECM designed and carried out the experimental work. EV performed the computacional analyses. FA participated in the design of the study. MS 
designed and carried out experiments, analyzed the data with the help of IS, and wrote the manuscript. All authors discussed the results and approved the final manuscript.

\section{Acknowledgements}

We thank Rodrigo Bermejo for critical reading of the manuscript and for providing strains. We also thank Orlando Domínguez for technical support with high throughput sequencing. This work was supported by the Spanish Ministry of Economy and Competitiveness [RYC-2009-04407 and BFU2010-20034 to M.S., BFU2011-28804 and Consolider-Ingenio CSD2007-00015 to F.A.]; Spanish Ministry of Economy and Competitiveness (Ramón y Cajal contract to M.S.); Spanish Ministry of Economy and Competitiveness (predoctoral fellowships to I.S. and E.C.); Consejo Superior de Investigaciones Cientificas (contract to E.V.). Funding for open access charge: Spanish Ministry of Economy and Competitiveness.

Received: 14 April 2014 Accepted: 10 September 2014

Published: 13 September 2014

\section{References}

1. Bell SP, Stillman B: ATP-dependent recognition of eukaryotic origins of DNA replication by a multiprotein complex. Nature 1992, 357:128-134

2. Remus D, Diffley JF: Eukaryotic DNA replication control: lock and load, then fire. Curr Opin Cell Biol 2009, 21:771-777.

3. Labib K: How do Cdc7 and cyclin-dependent kinases trigger the initiation of chromosome replication in eukaryotic cells? Genes Dev 2010, 24:1208-1219.

4. Santocanale C, Sharma K, Diffley JF: Activation of dormant origins of DNA replication in budding yeast. Genes Dev 1999, 13:2360-2364.

5. Wyrick JJ, Aparicio JG, Chen T, Barnett JD, Jennings EG, Young RA, Bell SP, Aparicio OM: Genome-wide distribution of ORC and MCM proteins in S. cerevisiae: high-resolution mapping of replication origins. Science 2001, 294:2357-2360.

6. Raghuraman MK, Brewer BJ, Fangman WL: Cell cycle-dependent establishment of a late replication program. Science 1997, 276:806-809.

7. Friedman KL, Brewer BJ, Fangman WL: Replication profile of Saccharomyces cerevisiae chromosome VI. Genes Cells 1997, 2:667-678.

8. Ferguson BM, Fangman WL: A position effect on the time of replication origin activation in yeast. Cell 1992, 68:333-339.

9. Vogelauer M, Rubbi L, Lucas I, Brewer BJ, Grunstein M: Histone acetylation regulates the time of replication origin firing. Mol Cell 2002, 10:1223-1233.

10. Aparicio JG, Viggiani CJ, Gibson DG, Aparicio OM: The Rpd3-Sin3 histone deacetylase regulates replication timing and enables intra-S origin control in Saccharomyces cerevisiae. Mol Cell Biol 2004, 24:4769-4780.

11. Knott SR, Peace JM, Ostrow AZ, Gan Y, Rex AE, Viggiani CJ, Tavare S, Aparicio OM: Forkhead transcription factors establish origin timing and long-range clustering in S. cerevisiae. Cell 2012, 148:99-111.

12. Knott SR, Viggiani CJ, Tavare S, Aparicio OM: Genome-wide replication profiles indicate an expansive role for $\mathrm{Rpd} 3 \mathrm{~L}$ in regulating replication initiation timing or efficiency, and reveal genomic loci of Rpd3 function in Saccharomyces cerevisiae. Genes Dev 2009, 23:1077-1090.

13. Pohl TJ, Brewer BJ, Raghuraman MK: Functional centromeres determine the activation time of pericentric origins of DNA replication in Saccharomyces cerevisiae. PLoS Genet 2012, 8:e1002677.

14. Raghuraman MK, Winzeler EA, Collingwood D, Hunt S, Wodicka L, Conway A, Lockhart DJ, Davis RW, Brewer BJ, Fangman WL: Replication dynamics of the yeast genome. Science 2001, 294:115-121.

15. Mantiero D, Mackenzie A, Donaldson A, Zegerman P: Limiting replication initiation factors execute the temporal programme of origin firing in budding yeast. EMBO J 2011, 30:4805-4814

16. Tanaka S, Nakato R, Katou Y, Shirahige K, Araki H: Origin association of SId3, Sld7, and Cdc45 proteins is a key step for determination of origin-firing timing. Curr Biol 2011, 21:2055-2063.

17. Looke M, Kristjuhan K, Varv S, Kristjuhan A: Chromatin-dependent and -independent regulation of DNA replication origin activation in budding yeast. EMBO Rep 2013, 14:191-198.

18. Kamimura Y, Tak YS, Sugino A, Araki H: Sld3, which interacts with Cdc45 (Sld4), functions for chromosomal DNA replication in Saccharomyces cerevisiae. EMBO J 2001, 20:2097-2107.

19. Aparicio OM, Stout AM, Bell SP: Differential assembly of Cdc45p and DNA polymerases at early and late origins of DNA replication. Proc Natl Acad Sci U S A 1999, 96:9130-9135.
20. Harbison CT, Gordon DB, Lee TI, Rinaldi NJ, Macisaac KD, Danford TW, Hannett NM, Tagne JB, Reynolds DB, Yoo J, Jennings EG, Zeitlinger J, Pokholok DK, Kellis M, Rolfe PA, Takusagawa KT, Lander ES, Gifford DK, Fraenkel E, Young RA: Transcriptional regulatory code of a eukaryotic genome. Nature 2004, 431:99-104.

21. Maclsaac KD, Wang T, Gordon DB, Gifford DK, Stormo GD, Fraenkel E: An improved map of conserved regulatory sites for Saccharomyces cerevisiae. BMC Bioinformatics 2006, 7:113.

22. Stevenson JB, Gottschling DE: Telomeric chromatin modulates replication timing near chromosome ends. Genes Dev 1999, 13:146-151.

23. Berbenetz NM, Nislow C, Brown GW: Diversity of eukaryotic DNA replication origins revealed by genome-wide analysis of chromatin structure. PLOS Genet 2010, 6:e1001092.

24. Eaton ML, Galani K, Kang S, Bell SP, MacAlpine DM: Conserved nucleosome positioning defines replication origins. Genes Dev 2010, 24:748-753.

25. Lipford JR, Bell SP: Nucleosomes positioned by ORC facilitate the initiation of DNA replication. Mol Cell 2001, 7:21-30.

26. Nieduszynski CA, Knox Y, Donaldson AD: Genome-wide identification of replication origins in yeast by comparative genomics. Genes Dev 2006, 20:1874-1879

27. Feng W, Collingwood D, Boeck ME, Fox LA, Alvino GM, Fangman WL, Raghuraman MK, Brewer BJ: Genomic mapping of single-stranded DNA in hydroxyureachallenged yeasts identifies origins of replication. Nat Cell Bio/ 2006, 8:148-155.

28. Yabuki $\mathrm{N}$, Terashima $\mathrm{H}$, Kitada $\mathrm{K}$ : Mapping of early firing origins on a replication profile of budding yeast. Genes Cells 2002, 7:781-789.

29. Alvino GM, Collingwood D, Murphy JM, Delrow J, Brewer BJ, Raghuraman MK: Replication in hydroxyurea: it's a matter of time. Mol Cell Biol 2007 27:6396-6406.

30. Vogelauer M, Wu J, Suka N, Grunstein M: Global histone acetylation and deacetylation in yeast. Nature 2000, 408:495-498.

31. Robyr D, Suka Y, Xenarios I, Kurdistani SK, Wang A, Suka N, Grunstein M: Microarray deacetylation maps determine genome-wide functions for yeast histone deacetylases. Cell 2002, 109:437-446.

32. De Piccoli G, Katou Y, Itoh T, Nakato R, Shirahige K, Labib K: Replisome stability at defective DNA replication forks is independent of $S$ phase checkpoint kinases. Mol Cell 2012, 45:696-704.

33. Santocanale C, Diffley JF: A Mec1- and Rad53-dependent checkpoint controls late-firing origins of DNA replication. Nature 1998, 395:615-618.

34. Shirahige K, Hori Y, Shiraishi K, Yamashita M, Takahashi K, Obuse C, Tsurimoto T, Yoshikawa H: Regulation of DNA-replication origins during cell-cycle progression. Nature 1998, 395:618-621.

35. Bell SP, Dutta A: DNA replication in eukaryotic cells. Annu Rev Biochem 2002, 71:333-374

36. Friedman $\mathrm{KL}$, Brewer $\mathrm{BJ}$ : Analysis of replication intermediates by two-dimensional agarose gel electrophoresis. Methods Enzymol 1995 262:613-627.

37. Kohzaki H, Murakami Y: Transcription factors and DNA replication origin selection. Bioessays 2005, 27:1107-1116

38. Diffley JF, Stillman B: Similarity between the transcriptional silencer binding proteins ABF1 and RAP1. Science 1989, 246:1034-1038.

39. Dominguez-Sola D, Ying CY, Grandori C, Ruggiero L, Chen B, Li M, Galloway DA, Gu W, Gautier J, Dalla-Favera R: Non-transcriptional control of DNA replication by c-Myc. Nature 2007, 448:445-451.

40. Minami H, Takahashi J, Suto A, Saitoh Y, Tsutsumi K: Binding of AIF-C, an Orc1-binding transcriptional regulator, enhances replicator activity of the rat aldolase B origin. Mol Cell Biol 2006, 26:8770-8780.

41. Irlbacher H, Franke J, Manke T, Vingron M, Ehrenhofer-Murray AE: Control of replication initiation and heterochromatin formation in Saccharomyces cerevisiae by a regulator of meiotic gene expression. Genes Dev 2005, 19:1811-1822.

42. Morse RH: RAP, RAP, open up! New wrinkles for RAP1 in yeast. Trends Genet 2000, 16:51-53.

43. Vujcic M, Miller CA, Kowalski D: Activation of silent replication origins at autonomously replicating sequence elements near the HML locus in budding yeast. Mol Cell Biol 1999, 19:6098-6109.

44. Doksani Y, Bermejo R, Fiorani S, Haber JE, Foiani M: Replicon dynamics, dormant origin firing, and terminal fork integrity after double-strand break formation. Cell 2009, 137:247-258.

45. Koerber RT, Rhee HS, Jiang C, Pugh BF: Interaction of transcriptional regulators with specific nucleosomes across the Saccharomyces genome. Mol Cell 2009, 35:889-902. 
46. Son J, Shen SS, Margueron R, Reinberg D: Nucleosome-binding activities within JARID2 and EZH1 regulate the function of PRC2 on chromatin. Genes Dev 2013, 27:2663-2677.

47. Ibarra A, Schwob E, Mendez J: Excess MCM proteins protect human cells from replicative stress by licensing backup origins of replication. Proc Natl Acad Sci U S A 2008, 105:8956-8961.

48. Shima N, Alcaraz A, Liachko I, Buske TR, Andrews CA, Munroe RJ, Hartford SA, Tye BK, Schimenti JC: A viable allele of $\mathrm{Mcm} 4$ causes chromosome instability and mammary adenocarcinomas in mice. Nat Genet 2007, 39:93-98.

49. Shivaswamy S, Bhinge A, Zhao Y, Jones S, Hirst M, lyer VR: Dynamic remodeling of individual nucleosomes across a eukaryotic genome in response to transcriptional perturbation. PLoS Biol 2008, 6:e65.

50. Lantermann A, Stralfors A, Fagerstrom-Billai F, Korber P, Ekwall K: Genomewide mapping of nucleosome positions in Schizosaccharomyces pombe. Methods 2009, 48:218-225.

51. Soriano I, Quintales $L$, Antequera F: Clustered regulatory elements at nucleosome-depleted regions punctuate a constant nucleosomal landscape in Schizosaccharomyces pombe. BMC Genomics 2013, 14:813.

52. Muller CA, Hawkins M, Retkute R, Malla S, Wilson R, Blythe MJ, Nakato R, Komata M, Shirahige K, de Moura AP, Nieduszynski CA: The dynamics of genome replication using deep sequencing. Nucleic Acids Res 2014, 42:e3.

53. Jiang C, Pugh BF: A compiled and systematic reference map of nucleosome positions across the Saccharomyces cerevisiae genome. Genome Biol 2009, 10:R109.

54. Lee W, Tillo D, Bray N, Morse RH, Davis RW, Hughes TR, Nislow C: A highresolution atlas of nucleosome occupancy in yeast. Nat Genet 2007, 39:1235-1244.

55. Brewer BJ, Lockshon D, Fangman WL: The arrest of replication forks in the rDNA of yeast occurs independently of transcription. Cell 1992, 71:267-276.

56. Bermejo R, Katou YM, Shirahige K, Foiani M: ChIP-on-chip analysis of DNA topoisomerases. Methods Mol Biol 2009, 582:103-118.

doi:10.1186/1471-2164-15-791

Cite this article as: Soriano et al:: Different nucleosomal architectures at early and late replicating origins in Saccharomyces cerevisiae. BMC Genomics 2014 15:791.

\section{Submit your next manuscript to BioMed Central and take full advantage of:}

- Convenient online submission

- Thorough peer review

- No space constraints or color figure charges

- Immediate publication on acceptance

- Inclusion in PubMed, CAS, Scopus and Google Scholar

- Research which is freely available for redistribution 